\title{
SWISH: A Shifting-Weight Interface of Simulated Hydrodynamics for Haptic Perception of Virtual Fluid Vessels
}

\author{
Shahabedin Sagheb, Frank Wencheng Liu, Alireza Bahremand, Assegid Kidane, Robert LiKamWa \\ Arizona State University, Tempe, AZ \\ ssagheb@asu.edu, fwliu1@asu.edu, abahrema@asu.edu, assegid.kidane@asu.edu, likamwa@asu.edu
}

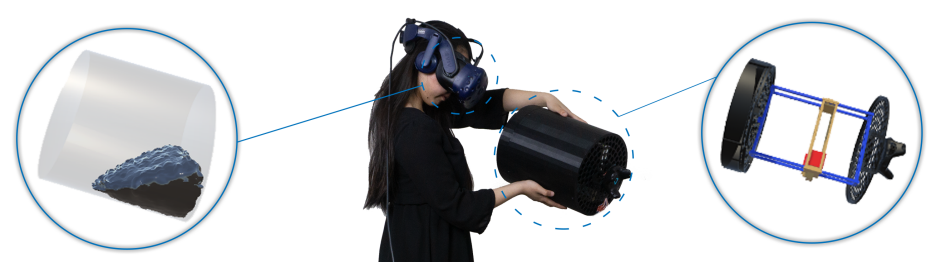

Figure 1. SWISH, a haptic feedback device capable of simulating fluid behavior in virtual reality or augmented reality. (Left) simulated virtual fluid in virtual reality, (Right) the mechanically actuated system that tangibly represents a fluid's center of gravity.

\begin{abstract}
Current VR/AR systems are unable to reproduce the physical sensation of fluid vessels, due to the shifting nature of fluid motion. To this end, we introduce SWISH, an ungrounded mixed-reality interface, capable of affording the users a realistic haptic sensation of fluid behaviors in vessels. The chief mechanism behind SWISH is in the use of virtual reality tracking and motor actuation to actively relocate the center of gravity of a handheld vessel, emulating the moving center of gravity of a handheld vessel that contains fluid. In addition to solving challenges related to reliable and efficient motor actuation, our SWISH designs place an emphasis on reproducibility, scalability, and availability to the maker culture.
\end{abstract}

Our virtual-to-physical coupling uses Nvidia Flex's Unity integration for virtual fluid dynamics with a 3D printed augmented vessel containing a motorized mechanical actuation system. To evaluate the effectiveness and perceptual efficacy of SWISH, we conduct a user study with 24 participants, 7 vessel actions, and 2 virtual fluid viscosities in a virtual reality environment. In all cases, the users on average reported that the SWISH bucket generates accurate tactile sensations for the fluid behavior. This opens the potential for multi-modal interactions with programmable fluids in virtual environments for chemistry education, worker training, and immersive entertainment.

Permission to make digital or hard copies of all or part of this work for personal or classroom use is granted without fee provided that copies are not made or distributed for profit or commercial advantage and that copies bear this notice and the full citation on the first page. Copyrights for components of this work owned by others than ACM must be honored. Abstracting with credit is permitted. To copy otherwise, or republish, to post on servers or to redistribute to lists, requires prior specific permission and/or a fee. Request permissions from permissions@acm.org.

UIST '19, October 20-23, 2019, New Orleans, LA, USA.

Copyright (C) 2019 Association of Computing Machinery.

ACM ISBN 978-1-4503-6816-2/19/10 ...\$15.00.

http://dx.doi.org/10.1145/3332165.3347870

\section{Author Keywords}

Ungrounded haptic feedback; virtual reality; fluid dynamics

\section{CCS Concepts}

-Human-centered computing $\rightarrow$ Human computer interaction (HCI); Mixed / augmented reality; Virtual reality; Haptic devices; User studies;

\section{INTRODUCTION}

One of the chief characteristics of a fluid is that it has no fixed shape. Instead, a fluid will continually reshape, subject to forces, including gravity, cohesion, adhesion, and contact with its vessel. Fluid simulation has made strides to emulate physical fluid properties in virtual software, enabling realistic visual rendering of fluids in simulations and games for scientific, educational, and entertainment scenarios. Fluid simulation packages especially enhance virtual reality (VR) or augmented reality (AR) environments, wherein users can handle a physical vessel filled with virtual fluid.

However, little has been done to render tangible sensations associated with the handling of fluid vessels. As a fluid moves, the center of gravity $(\mathrm{CoG})$ of the vessel shifts, substantially altering the moment of force on the handling of the vessel. In current VR/AR environments, physical vessels do not physically represent this shift. As a result, while virtual fluids in vessels may look realistic, they do not feel realistic. The aim of this work is to create faithful sensations of fluid handling interactions by shifting the CoG of the vessel in realtime.

To this end, we present SWISH, a Shifting-Weight Interface for Simulated Hydrodynamics. As illustrated in Fig. 1. SWISH uses a cylindrical motorized system to relocate the center of gravity of a physical vessel. The physical vessel is tracked in the 3D space of the virtual environment, integrated with 
NVIDIA Flex ${ }^{1}$, an off-the-shelf virtual fluid simulation framework. Based on the fluid dynamics of the simulation, our software translates fluid particle positions to vessel center of gravity, which the software further translates to SWISH motor commands. The SWISH motor commands are wirelessly transmitted via Bluetooth to the physical SWISH vessel, whereupon our mechanical device appropriately relocates the CoG. This system thus creates tangible sensations necessary to physically handle virtual fluids.

Notably, the SWISH system allows its virtual fluids to be expressively programmable. The virtual fluid simulation framework provided by NVIDIA Flex allows software developers to define varying volumes and viscosities of fluids, as well as cohesion and adhesion properties of the fluid particles. Such properties can be altered at runtime, allowing fluids to transform their characteristics to match accurate representations of chemical reactions or present a whimsical materiality of fictional substances. Operating on this expressive fluid simulation framework, SWISH naturally enables a haptic realization of these programmable fluids.

In this paper, we discuss the design of SWISH mechanisms, with attention to two primary challenges of: (i) actuating center of gravity shifts through a motorized apparatus, and (ii) coupling virtual simulation with physical actuation. We design our mechanisms with attention on their ease to scale for broad populations through accessible software frameworks and prototypable hardware mechanisms. To encourage broader use, we will release our designs as open source on an online repository at the time of publication.

Our user study evaluation of 24 users finds that users experienced realistic tactile perceptions with the SWISH bucket, largely agreeing with the statement "Tactile sensation of handling the liquid using this haptic input device was accurate". Through the study, we find that users find SWISH to be similar in fidelity to a vessel containing real fluid, and to outperform an empty vessel with no fluid. As SWISH does not rely on the inconvenient presence of real fluids - reducing risks of spills and enables fluids with programmable volumes and viscosities, it opens a wide range of possibilities towards future use cases, enhanced with a tactile perception of fluid dynamics.

Altogether, we present the following contributions:

- Designing SWISH, a tactile interface to mimic the centerof-gravity shifts of fluid vessels through responsive motor actuation integrated with a virtual fluid simulation.

- Implementing motor actuation techniques to represent fluids with high fidelity and expressiveness, and

- Quantifying the effectiveness of the SWISH haptic interface through a VRUSE-based user evaluation across 24 users.

\section{Situational use cases for VR/AR fluid vessels}

Natural liquids exist abundantly; we interact with various types of liquids on a daily basis. During everyday interactions, people perform multiple tests to evaluate various characteristics of liquid vessels, among which are shaking, tilting, swaying,

\footnotetext{
$\overline{{ }^{1} \text { https://developer.nvidia.com/flex }}$
}

and other fluid interactions, as shown in Fig. 2. For instance, one might gently sway a cooking pot to evaluate whether there is some water left in it. Thus, realistic haptic feedback is especially important for simulation-based training and evaluation of fluid-handling practices, as the training will need to reflect real experiences for successful skill acquisition. While humans are familiar with the qualities of household liquids, e.g., water and cooking oil, users can perform multiple tests to identify and understand foreign liquids. Successful fluid haptic simulation would yield a new class of affordances for fluid-based media.

Employers can adopt VR/AR fluid systems for training/evaluation situations involving hazardous fluids. For example, to limit untrained exposure to harmful chemicals, the proposed augmented fluid technology can allow workers to interact with augmented fluid in a vessel that simulates fluid properties of hazardous materials, e.g., color, viscosity, reactivity. Virtual fluid materials must realistically react to workplace illumination and physical manipulation to create representative training scenarios to improve worker training efficacy and safety. Furthermore, the fluid system can observe and track fluid handling tasks for continuous worker evaluation, e.g., tracking safe practices, efficient material use, and worker proficiency. Using augmented fluids in dangerous or hazardous situations comes with the added advantage of avoiding disastrous consequences if a mistake is made during skill training.

Moreover, VR/AR fluid systems enable workers to pursue open exploratory experimentation with fluid handling. Most fluid combinations are not separable once mixed and many combinations initiate reactions with time limits on processing and use. For example, mixing silicone rubber or epoxy requires a homogeneous polymer mixture, but there is a limited time for application of these costly materials. Timing is critical; there is risk of product failure if these materials are applied too early or too late. We imagine workers being enabled to rehearse a variety of processes to identify optimal physical layouts, tools, vessels, and to gain kinesthetic experience with specific fluid-based activities without material waste.

VR/AR fluid systems also enable workers to travel with portable remote laboratories, enabling augmented testing from homes and hotel rooms. Complete with the ability to interact with the fluids through vessel interactions, e.g., swirling and pouring, workers can rapidly interact with simulated fluid experiments. For such interaction, it is important for the augmented system to replicate visual-tactile interactions to represent experimental conditions. Workers can also use mixed reality fluid systems for immersive visualization of additional layers of information. For example, through the AR or VR headset, the worker can view temperature gradients, density, turbulent flow, laminar flow, or other fluid properties spatially overlaid onto/into the virtual fluid. With overlays of transparent colors, workers can see catalyzed reactions. The augmented vessels provide workers with rich real-time agency over view and manipulation of the phenomena. 


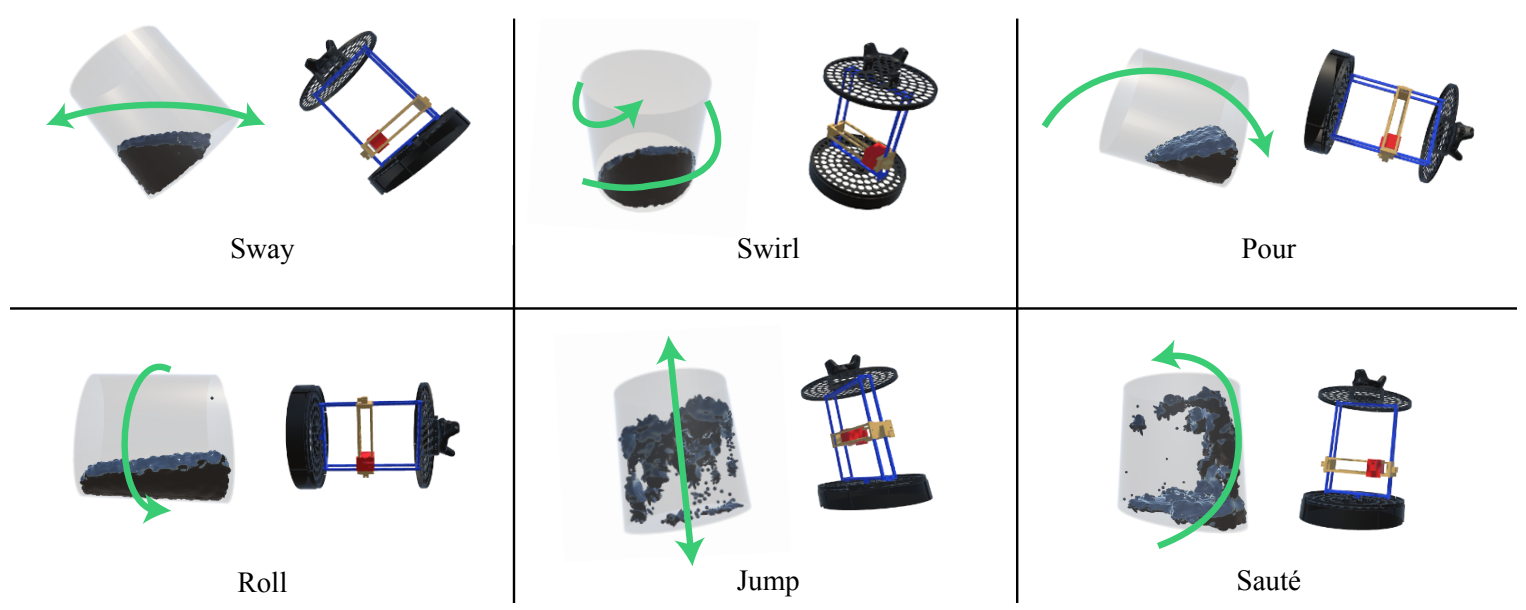

Figure 2. Six different actions for interacting with the SWISH haptic input system

\section{RELATED WORK}

\section{Wearable and Handheld Haptic Devices}

While commercial and academic research works have enabled rich visual integration of digital and physical objects, tangible integration has largely been focused on simulating the sensations of interacting with rigid objects using vibrotactile [6, 16], exoskeleton gloves [4, 29, 8], robotic arms [1], passive proxies $[9,26,18]$, and grounded devices $[14,20]$, with a few ungrounded systems $[15,27,3]$.

\section{Weight-shifting Haptic Devices}

As early as 1965 the effective stimulus in sensitivity to torque has been studied. Woodruff and Helson [24] investigated the effect of shifting the location of a mass along a rod and reported increased perceived heaviness when torque increases. Researchers have leveraged this phenomenon to design mass shifting devices. TorqueBAR [19] and Shifty [27] alter internal weight distribution to alter perception of virtual objects.

Current haptic systems present sensations related to virtual mass, force, and texture. Gravity Grabber presents perception of mass via deformation on fingerpads [15], while FinGAR delivers glove-based electrical and mechanical stimulation for tactile feedback [25]. Others have proposed haptic feedback mechanisms that use mechanical torque cues [22], [23], texture simulation [3] and force [4], [29]. Open systems addressing the change in weight and volume of an object have been proposed [17]. Systems based on haptic illusion have also been designed that provide a tactile sensation of mass [7].

\section{Grounded Haptic Devices}

Closely related to our work, some grounded devices present nuances of fluid dynamics. Cirio et al. [5] incorporated a commercially available 6 DoF haptic device to present physical sensations of interacting with solids, deformable objects, and multi-state fluids. Using an end-effector as a proxy (e.g. a spoon), the user is able to interact with a simulated liquid content and feel the force feedback. Vines et al. [21] present a system that allows the user to stir a virtual fluid container using a similar commercially available device. Zhang et al. [28] use the same device to provide haptic sensation related to touch, push and sensing various stationary and moving fluid. While these grounded systems provide a wider range of forces and torques that can be rendered in VR, their setup and use case scenarios are limited to stationary workstations. The proxies used are external objects that interact with the simulated liquid (e.g. pushing the surface of water using a pen-shaped proxy).

Our work extends these solutions, addressing challenges towards ungrounded perception of fluid movement in vessels. Also, we present a system that is scalable and can be made with off-the-shelf components and 3D printed parts.

\section{SWISH DESIGN CONSIDERATIONS}

The development and use of the SWISH vessel poses the following design considerations:

- Fluid fidelity. SWISH should quickly and accurately reposition the $\mathrm{CoG}$ to faithfully represent the forces and moments associated with virtual fluids.

- Fluid expressiveness. SWISH should service different virtual fluid volumes and viscosities.

- Accessibility/Affordability. The physical structure and electronic hardware of SWISH should be built from off-theshelf components and/or be constructed with consumergrade digital fabrication tools and materials.

- Modularity/Scalability. The mechanisms underlying SWISH should have an adaptive and scalable design that can be configured for various vessel shapes and sizes

\section{SWISH IMPLEMENTATION}

Built on these considerations, our implementation of SWISH uses the combination of: (i) a prototypable mechanical actuation framework to actuate $\mathrm{CoG}$ relocation by moving an active mass, and (ii) a software virtual-to-physical integration framework to integrate virtual fluids from virtual reality and augmented reality settings into physical environments. Fig. 3 illustrates an overview of the integrated SWISH system.

We designed SWISH with a special attention to the use of standardized parts, modularity, and reusable design. In doing 


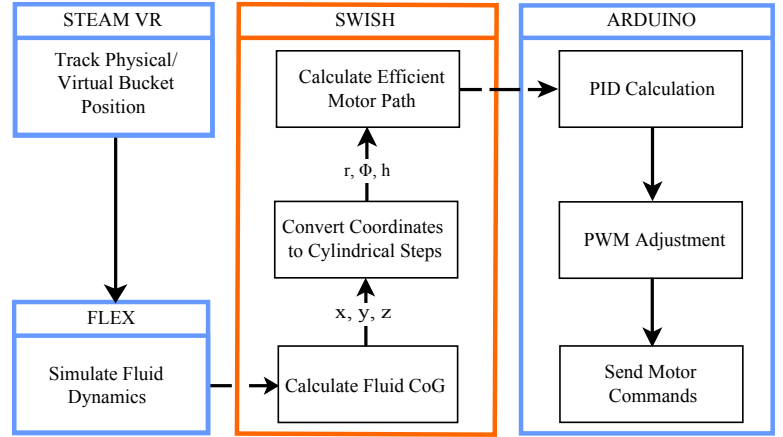

Figure 3. End-to-end system block diagram

so, we emphasize the use of openly available software and hardware tools to provide a design accessible to the maker community. Our mechanical actuation system uses a combination of 3D printable parts and off-the-shelf electronic components for CoG actuation. Our virtual-to-physical integration framework uses NVIDIA Flex for fluid particle simulation, Unity Game Engine for virtual scene composition and rendering, and the HTC Vive Lighthouse system for virtual-physical vessel positioning. Furthermore, the actuation mechanism and the structure of SWISH are scalable to fit inside a variety of symmetric vessel housings.

\section{Mechanical Actuation System}

As presented in Fig. 4, SWISH's mechanical system has an internal motorized structure inside of an external stationary housing. This section covers design aspects for the motorized structure, including ensuring motor actuation fidelity and efficient motor movement patterns.

The rotational symmetry of the cylindrical coordinate system aligns with typical vessel shapes. As such, it is convenient to provide structural support for the three cylindrical axes. We designed a rack-and-pinion mechanism based on gantry system actuation that sits inside of the cylindrical volume within a vessel. A motor at the base of the central axis controls azimuthal $(\phi)$ changes to the CoG. A pair of elevation motors on the central axis controls the height $(h)$. Finally, a motor along a radial arm relocates a weighted mass to control the radius $(r)$ of the $\mathrm{CoG}$.

\section{PID-based motor control for movement fidelity}

The chief component of authentic simulation is reliable path following. To this end, we implemented a proportional-integral-derivative (PID) control loop feedback system to precisely control motor positions. The microcontroller adjusts the PWM signal and sends step commands to the motor drivers at a rate of 100 commands per second. We calculate PID coefficients using the empirical step response to setpoint method [2], while the entire SWISH mechanism rests on a desk. During calculation, the active mass was mounted on the actuation system to account for load disturbance.

\section{Radius polarity selection for efficient movement commands}

The SWISH software system makes motor control command decisions to prioritize smooth and efficient movement between motor positions. Because of the rotational symmetry of the
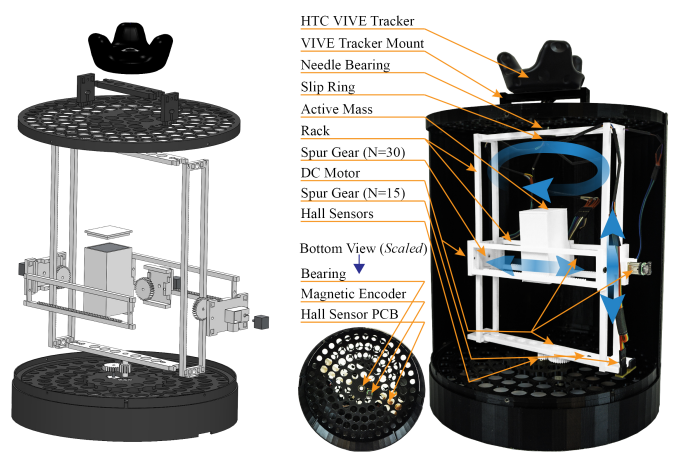

Figure 4. Mechanical structure and actuation mechanism

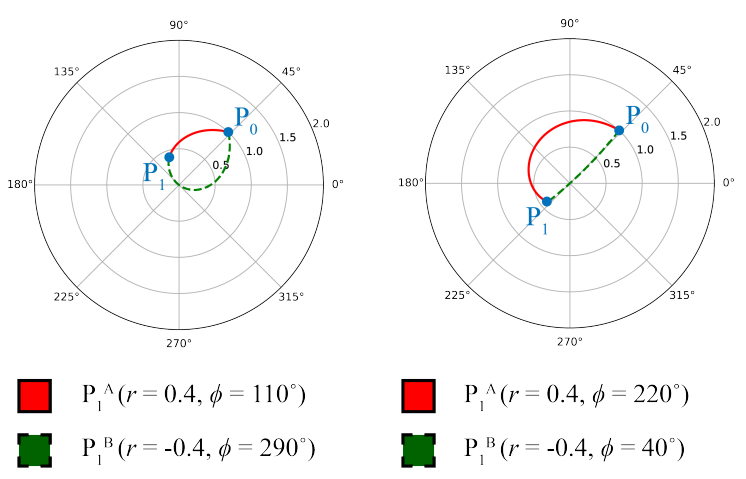

Figure 5. There are two equivalent $(\mathrm{r}, \phi)$ representations for anticipated target position(s). Based on the start position and end position, one path requires less motor movement than the other.

cylindrical coordinate system, each $\mathrm{CoG}$ position has two equivalent motor positions: $(r, \phi)$, and $(-r, \phi \pm 180)$. For every sequential position command, it may be more favorable to choose a path towards one motor position over the other.

Fig. 5 shows potential scenarios for motor movements from $P_{0}$ to $P_{1}$. On the left side of the figure, we see a case where it is favorable to move to the position with positive radius $\left(P_{1}^{A}\right)$, creating less movement than the path to the position with negative radius $\left(P_{1}^{B}\right)$. On the right side of the figure, we see the reverse is true; having the mass travel along the radial arm to use a negative radius position $\left(P_{1}^{B}\right)$ results in less movement.

Thus, to minimize motor movement and provide a faster actuation of active mass, SWISH makes a decision by comparing the number of radial motor steps and azimuthal motor steps required to achieve either position. SWISH uses a minimax algorithm, prioritizing whichever position pair requires fewer maximum motor steps among radial and azimuthal motors.

\section{Rapid prototyping details}

All of the 3D printed components are printed using the Stratasys Dimension 1200es (FDM technology) 3D printer, using ABS material. A stationary housing encapsulates the entire cylindrical mechanism, with diameter $248 \mathrm{~mm}$ and height $262 \mathrm{~mm}$. The active mass moved by the actuation mechanism weighs $561 \mathrm{~g}$. The entire SWISH vessel weighs $1477 \mathrm{~g}$.

We designed a custom Arduino shield that houses motor drivers, resistors, and connectors. The Arduino, Bluetooth 


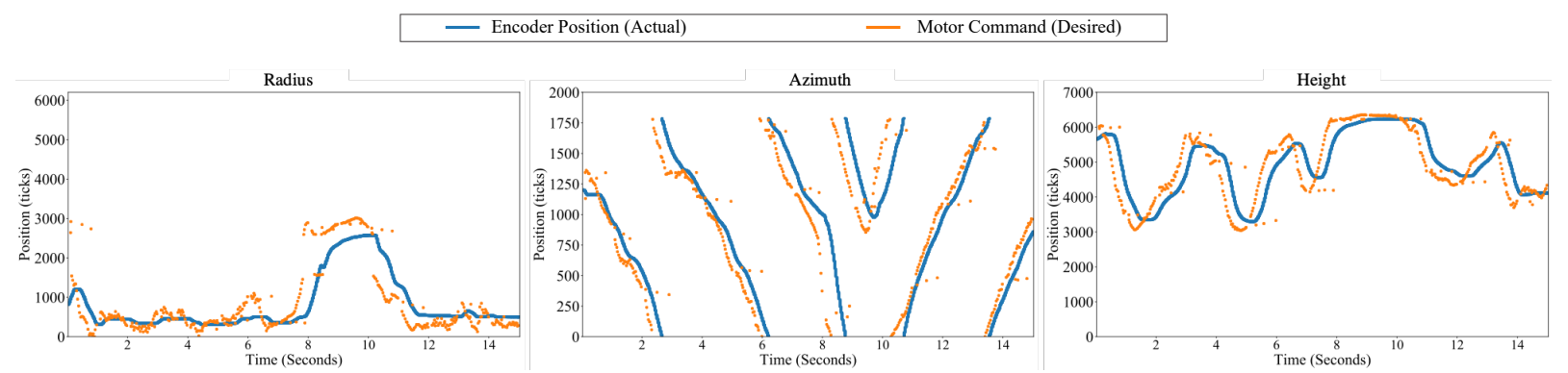

(a) Liquid One (Low Viscosity)
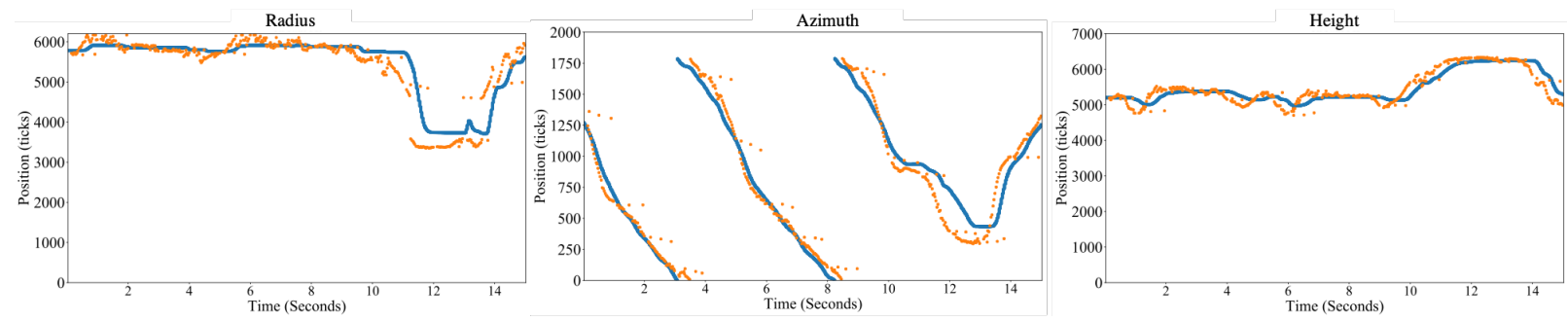

(b) Liquid Two (High Viscosity)

Figure 6. Received motor commands (orange dots) and motor position (blue line) for the "Swirl" action. (Other actions are in the Appendix).

module, and motors are powered using an external power supply $($ Voltage $=6 \mathrm{~V}$, Current $=1.5 \mathrm{~A})$. During peak performance we measured that the current did not surpass $1.4 \mathrm{~A}$.

\section{Digital-physical integration details Spatial tracking}

As shown in Fig. 4, we use a custom-printed mount to attach an HTC Vive Tracker to the vessel for augmented tracking of the vessel's position and rotation. The SteamVR framework for the Unity Game Engine reports the relative physical coordinates of the Vive Tracker in the environment. This achieves an augmented tracking performance of 30 frames per second.

\section{NVIDIA Flex integration for Unity}

Our SWISH implementation uses NVIDIA Flex to simulate fluid dynamics. Flex simulates particle interactions using Unified Particle Physics [13], and Position-Based Fluids [12] in real-time. This allows fluid particles to interact with each other and with the rigid bodies in the virtual environment, emulating properties of cohesion, surface tension, adhesion, and other fluid properties. The virtual fluid in the vessel thus operates with similar characteristics to physical fluids.

The fluid simulation created by the computational engine uses cartesian world-space virtual scene coordinates. As discussed before, the physical bucket motors are designed based on cylindrical coordinates. SWISH computes the translation between these coordinate systems using a sequence of mappings as shown in Figure 3. First, Flex calculates the position of each particle in the cartesian "world coordinate system" of the virtual space. SWISH averages the particle positions to compute the location of the $\mathrm{CoG}$, computing its displacement relative to the bucket's origin. SWISH then converts the displacement vector from cartesian to cylindrical coordinates. The polar position vectors are linearly scaled to represent movement commands for DC motor positions. SWISH then employs the

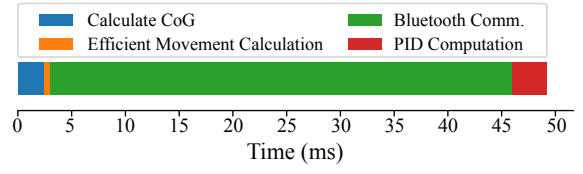

Figure 7. Latency of SWISH system components

aforementioned radius polarity algorithm to select an efficient motor destination. The resulting position values are sent to the Arduino as set point commands for PID calculation.

Flex fluid parameters control the behavior of the fluid dynamics of particles inside the virtual scene. In this paper we focus on particles with high and low viscosities, roughly matching the fluid properties of water and vegetable oil. SWISH will naturally respond to the change in viscosity; for viscous fluids, the simulation will report a less active sequence of $\mathrm{CoG}$ positions. SWISH will operate the actuation mechanism accordingly.

We designed SWISH to maximize the effect of CoG shift. The shape of the mass effector was designed to have a smaller profile along the radius axis to allow higher distance of travel along this axis. A longer distance of travel along radius has a pronounced effect on the CoG shift. The height of the mass effector was maximized to highest possible to hold the largest amount of mass that the specific motors can actuate with sufficient torque.

\section{TECHNICAL EVALUATION}

Quick and accurate actuation is integral to the fidelity of the haptic response. To evaluate the software/hardware integration, we performed six characteristic actions, as represented in Fig.2: Sway, Swirl, Pour/Flip, Roll, Jump, and Sautée. From these, we report end-to-end latency and CoG accuracy. 
Table 1. Mean path error

\begin{tabular}{|c|c|c|c|}
\hline Action & Azimuth Motor & Radius Motor & Height Motor \\
\hline Sway & $1.131 \%$ & $1.174 \%$ & $1.307 \%$ \\
\hline Swirl & $0.066 \%$ & $0.905 \%$ & $0.145 \%$ \\
\hline Pour & $4.299 \%$ & $0.525 \%$ & $0.291 \%$ \\
\hline Roll & $0.736 \%$ & $1.235 \%$ & $1.065 \%$ \\
\hline Jump & $7.794 \%$ & $0.949 \%$ & $1.516 \%$ \\
\hline Sautée & $10.573 \%$ & $1.376 \%$ & $1.677 \%$ \\
\hline
\end{tabular}

\section{Sample Rate and Latency.}

To understand the responsiveness of the SWISH system, we measure the sample rate and latency of the motor commands as they are generated through the software-hardware integration system. We record timestamps at: (i) the time the particles are generated, (ii) the time the CoG is calculated, (iii) the time the efficient motor command is calculated, (iv) the time the motor command was received by the Arduino over Bluetooth, and (v) the time the PID-controlled motor command is sent to the motor driver. This allows us to compute the sample rate and latency of each component of the SWISH system.

The overall sample rate of SWISH maintains a consistent $30 \mathrm{~Hz}$, limited only by the update rate of the Flex particle system. As all SWISH components are pipelined with one another, the update rate is limited only by the frame-dependent generation of particles. However, SWISH does carry a latency of approximately $50 \mathrm{~ms}$, largely due to the latency of Bluetooth transmission. Indeed, as shown in Fig. 7, while $\mathrm{CoG}$ calculation, Efficient Movement Calculation and PID computation each require less than $3 \mathrm{~ms}$, the Bluetooth communication takes tens of milliseconds. In the future, we plan to resolve this latency by using wireless connections with higher reliability and lower latency, e.g., the $<5$ ms latency of $\mathrm{WiFi}$ modules.

\section{Accuracy}

We evaluated the accuracy of the mechanical system in following the virtual fluid's CoG in multiple test cases. Each of the six characteristic actions was repeated three times. Then, the location of SWISH's CoG was compared against the simulated fluid's CoG. Notably, because of the use of encoder-based PID control, errors do not accumulate; the SWISH Arduino maintains absolute positioning of each motor axis.

As shown in Fig. 6 the SWISH mechanism is able to closely follow the prescribed path for the Sway motion, though the actual CoG position is bounded by maximum motor speed capabilities against motor torque loads. The remainder of the motions are shown in the Appendix.

In Table 1, we list the path error of the SWISH mechanism, calculated via cross-correlation of the motor's $\mathrm{CoG}$ path with the prescribed path of the fluid's CoG. As seen in the table, each SWISH motor maintains a small mean path error $(<1.3 \%)$ for Sway, Swirl, and Roll actions. The radial SWISH motor begins to deviate for the Pour/Flip action, with a $4.3 \%$ mean path error. The large motions of the Jump and Sautée actions created difficulty for the radial SWISh motor to match the erratic patterns, resulting in radius path errors of $7.8 \%$ and $10.6 \%$ respectively. Altogether, across the non-Jump/Sautée actions and motors, SWISH is capable to actuate the vessel's
CoG to follow the path of the virtual fluid's CoG with a mean path error of $1.0 \%$ for each motor axis.

\section{Size and weight of the active mass}

We designed SWISH to maximize the effect of CoG shift. The shape of the mass effector was designed to have a smaller profile along the radius axis to allow travel distance along this axis. A longer distance of travel along radius supports a larger range of effects on the CoG shift. The height of the mass effector was maximized to hold the largest amount of mass that the specific motors can actuate with sufficient torque.

\section{USER STUDY}

We designed a user study to evaluate the perceived realism of SWISH in providing the tactile sensations of interacting with virtual fluids. Our hypothesis is that SWISH outperforms an incongruent solid proxy and closely matches a congruent fluid proxy. To this end, we designed a virtual scene, including 3 virtual-physical vessel buckets and 2 virtual liquids with different simulated viscosities. Fig. 8 displays the scene.

Through HTC Vive Lighthouse trackers, we mapped the virtual buckets to 3 physical buckets in the study room. In the virtual world, the buckets were identical in shape and size. Similarly, in the physical world, all of the buckets had the same size, shape, and weight (1477 g). However, the physical buckets exhibited different contents: \{Bucket 1 with SWISH mechanism, Bucket 2 with Water (congruent fluid proxy), Bucket 3 Empty (incongruent solid proxy) .

We mapped the location of the table so that users could pick up and set down the buckets on a known surface. During the study, a proctor communicated with participants through headphones via an external microphone. Throughout the interaction participants could hear a white noise through the headphones, which damped environmental noises (but was not wholly effective in masking all motor noise).

\section{Participants}

We advertised the study via email. After screening, we selected 24 participants ( 4 female, 20 male) with ages ranging betweeen 19-33 (Mean $(M)=24$, Standard Deviation $(\mathrm{SD})$ $=3.77$ ). The purpose of screening was to identify and mitigate any pre-existing conditions that would cause discomfort while using VR headsets. 21 participants reported that they had used VR before and 12 of them had used a haptic controller in VR. Participants entered a raffle to win a $\$ 10$ gift card.

\section{Data Collection}

After signing a consent form, participants answered an online demographic questionnaire. During the study we used a modified VRUSE questionnaire [11] inside the virtual scene to record users' feedback, as shown in Fig. 8. The same questionnaire was used for the two phases of evaluation, discussed below. After the study, participants answered an online questionnaire with specific questions regarding performance of SWISH with a mix of multiple choice and open-ended questions. Table 2 presents each questionnaire and its link to VRUSE. The entire study took roughly an hour. 


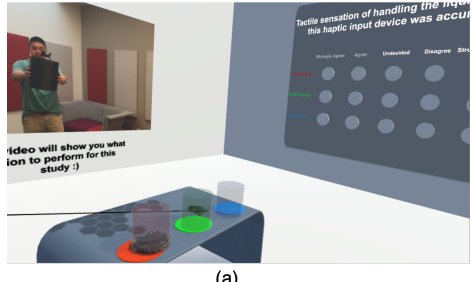

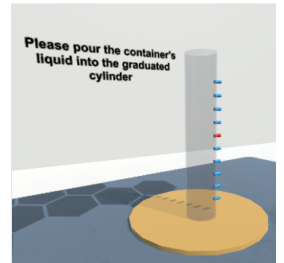

(b)
Figure 8. (a) User study VR scene composition. (b) Graduated cylinder used in pouring study.

\section{Phase 1 - Closed bucket interactions}

\section{Task and Procedure}

While wearing the VR headset, participants were tasked with using each bucket to perform the six characteristic actions from Fig. 2: Sway, Swirl, Pour/Flip, Roll, Jump, and Sautée. To prepare for each action, participants watched a 5-second video tutorial of how to perform the action in the virtual scene and repeated the action for each bucket. We used the Latin square design [10] to counter-balance the order of the buckets.

After each action, users were prompted to respond to a statement in the scene: "Tactile sensation of handling the liquid using this haptic input device was accurate". Participants could answer the questionnaire by pointing Vive controllers to choose how they felt about the statement: Strongly Disagree, Disagree, Undecided, Agree, Strongly Agree. Participants were allowed to repeat actions and change responses before submission. We performed the first part of Phase 1 with a virtual liquid with a low simulated viscosity. After completion of all actions, we switched the virtual liquid to another with a higher simulated viscosity. For the entire duration of this phase participants wore the headset for 20 minutes.

\section{Results}

Fig. 9 displays the aggregate results of the user responses. For all actions, users on average agreed that both the SWISH bucket and the water bucket gave appropriate tactile sensations of handling the liquid. For non-Jump/Sauté actions with the empty bucket, users were undecided about the accuracy of the tactile sensation of the empty bucket. For Jump/Sauté actions with the empty bucket, users reported that they disagreed that the water bucket gave accurate tactile sensations.

While performing the actions with the low viscosity liquid, SWISH outperforms the solid empty bucket in all actions and comes close to providing comparative sensations with the water bucket. For the jump task, SWISH $(\mathrm{M}=3.75, \mathrm{SD}=$ $0.98)$ was rated closest to the water bucket $(\mathrm{M}=3.79, \mathrm{SD}=$ $1.35)$ in providing an accurate tactile sensation.

As shown in Fig. 9 for the virtual liquid with high viscosity, users reported similarly positive feedback while using SWISH. However, the water bucket felt slightly less accurate when being paired with the viscous liquid. For this phase SWISH outperformed the solid empty bucket again. For the jump task, SWISH $(\mathrm{M}=3.79, \mathrm{SD}=1.25)$ outperformed the bucket filled with water $(\mathrm{M}=3.12, \mathrm{SD}=1.29)$ in providing an accurate tactile sensation. In sauté action, SWISH was perceived as providing a similar tactile sensation to the water bucket $(\mathrm{M}=$
3.91, $\mathrm{SD}=0.71$, Bucket with water: $\mathrm{M}=3.91, \mathrm{SD}=1.13$ ). SWISH was perceived positively in Flip and Roll actions.

\section{Discussion}

The data supports our hypothesis that SWISH provides realistic tactile sensations compared to an incongruent solid proxy (empty solid bucket). For both virtual liquids, users felt that the solid mass bucket was unable to provide an accurate and realistic sensation of handling a fluid. Meanwhile, users agreed that SWISH did provide accurate tactile sensations.

However, SWISH fell short of matching the strength of user response toward the congruent fluid proxy (water bucket). Even when the viscosity of simulated liquid was higher than real water in four out of six actions, users seemed to slightly prefer the bucket filled with water over SWISH. Analyzing the responses and cross-referencing with testimonials questionnaire revealed that several factors were involved in this finding. SWISH in its current form is connected to the Arduino using several wires from the top and bottom of the housing. While handling SWISH for each actions, some users reported difficulty in handling the bucket, due to wires obstructing the flow of action. Also, in the post-study evaluation, users mentioned that they thought the simulated low viscosity liquid was in fact supposed to appear and feel like water and interacting with it using a bucket filled with water was considered more natural. (This was despite the fact that proctor referred to the simulated liquid as liquid one and liquid two). Furthermore, some users reported that the sounds of the real water assisted in the realistic multi-modal perception of the virtual fluid.

\section{Phase 2 - Open bucket interactions Task and Procedure}

In the second phase of our study we focused on performing a pouring task with an open bucket. After a short break, and wearing the headset again participants entered another scene with a similar composition as the first one, with the addition of a graduated cylinder, seen in Fig.8b. We asked the participants to pour a virtual liquid inside each bucket to the graduated cylinder until it reached a red mark. A separate Latin square design was used to counter-balance the order of the buckets. This task was repeated for all buckets and participants had the option of repeating the task if they chose to.

We asked users to perform the first part of Phase 2 with a virtual liquid with low viscosity. After the completion of the pouring action with all three buckets, we switched the virtual liquid to a higher viscosity. After performing each pouring action, participants were prompted to respond to the same statement in the virtual scene: "Tactile sensation of handling the liquid using this haptic input device was accurate". We also recorded the virtual liquid's level poured into the graduated cylinder for each bucket and simulated liquid.

\section{Results}

Fig. 10 shows the aggregate users' responses to the accuracy of tactile sensation while performing this task. For both scenes, users on average reported that both SWISH and the water bucket felt accurate, while remaining undecided about the empty bucket. In the first scene with the low viscosity liquid, the bucket filled with water was rated more accurate, 


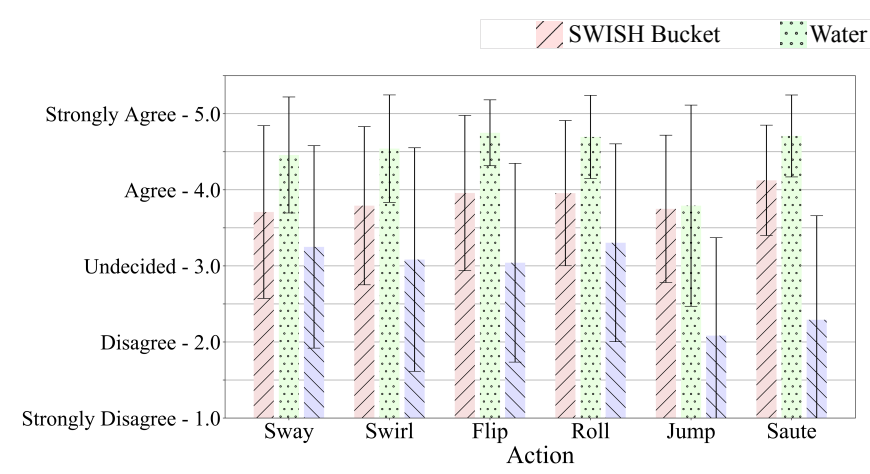

(a) Liquid One (Low viscosity)

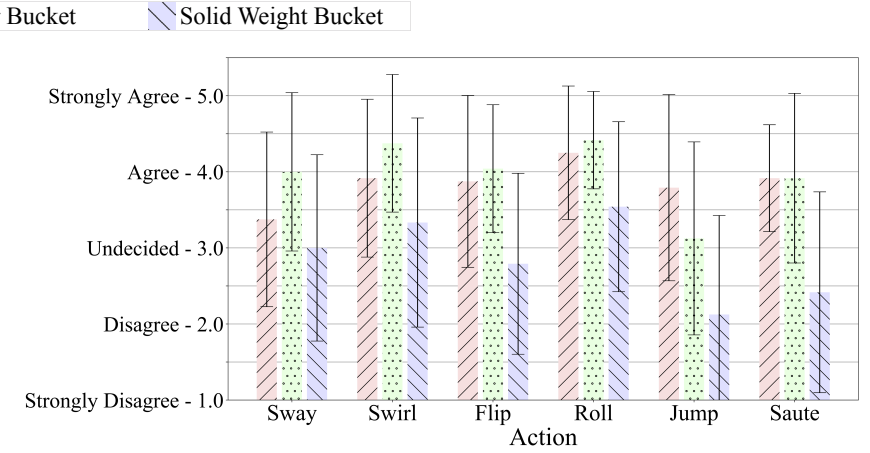

(b) Liquid Two (High viscosity)

Figure 9. User study responses for fidelity of actions with virtual fluid in closed bucket for SWISH (Red slant-up), Water (Green dots), and Solid (Blue slant-down).

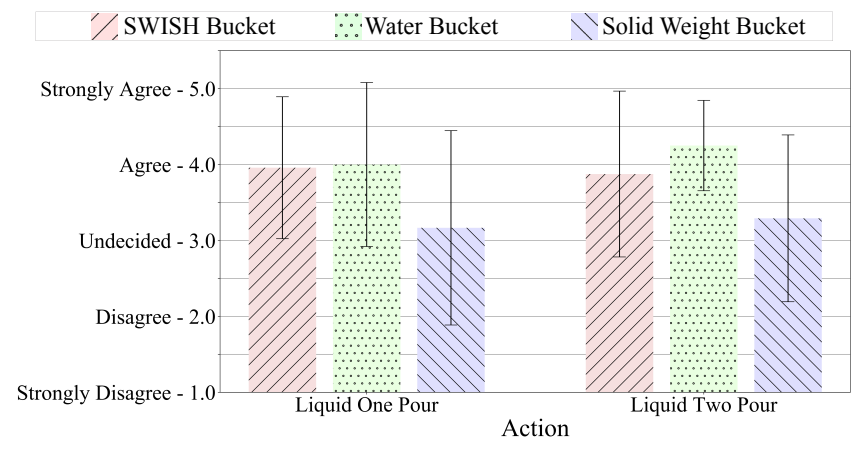

Figure 10. User-reported fidelity of pouring from open bucket. SWISH (Red slant-up), Water (Green dots), and Solid (Blue slant-down).

followed by SWISH $(\mathrm{M}=3.95, \mathrm{SD}=0.95)$, and the solid empty bucket $(\mathrm{M}=3.16, \mathrm{SD}=1.3)$. In the second scene with raised viscosity, SWISH $(\mathrm{M}=3.87, \mathrm{SD}=1.11)$ performed close to the bucket filled with water $(\mathrm{M}=4.25, \mathrm{SD}=0.60)$ and outperformed solid empty bucket $(\mathrm{M}=3.29, \mathrm{SD}=1.12)$.

We designed the graduated cylinder to hold 650 particles when filled up to the red notch. In the first scene with low viscosity simulated liquid, users were most successful when using the water bucket $(\mathrm{M}=671.25, \mathrm{SD}=84.23)$ followed by the solid empty bucket $(\mathrm{M}=692.83, \mathrm{SD}=89.27)$ and then SWISH $(M=714.46, S D=102.79)$. In the second scene with the higher viscosity simulated liquid bucket filled with water was again successful in assisting the users in performing this task $(\mathrm{M}=669.875, \mathrm{SD}=92.11)$ followed by SWISH $(\mathrm{M}=718.71, \mathrm{SD}=99.01)$ and solid mass empty bucket $(\mathrm{M}=724.91, \mathrm{SD}=132.46)$.

\section{Discussion}

This phase provided further support for our hypothesis towards SWISH's ability to provide accurate tactile sensation. However, our numbers report that SWISH did not enhance the precision of handling liquid in task related actions. This may have been due to flaws in our experimental setup. Participants mentioned that the location of the graduated cylinder was not convenient for the pouring task and obscured their proper view to perform the task more precisely which explains the deviation from the ideal level. In some cases, participants also reported that the sound and vibration of SWISH motors gave distracting feedback from the vessel. This study provides strong motivation towards the further development of smoother and/or shock-absorbed motor actuation.

\section{User Feedback}

As shown in Table 2, users responded positively to using SWISH as a haptic interface $(\mathrm{M}=4.17, \mathrm{SD}=0.76)$. Analyzing responses to the testimonials revealed that responsiveness of SWISH had a major impact in improving tactile perception; Participant 9 mentioned that: "It was an interesting experience. In my opinion it was extremely responsive to my movements, and I was impressed with its detection of my actions." Participant 11 wrote that: "It gave an accurate example of the different tests and which one was the most accurate as far as feelings went." Others mentioned that SWISH was effective in: providing a realistic sensation of weight (Participants 1,3) and its distribution (Participants 10,7), high responsiveness (Participants 09, 13, 14), smooth movements of the motorized mechanism (Participant 15), adaptivity to different viscosity (Participant 17) Users also reported some shortcomings related to both hardware and software. These included computational lag in the particle simulation itself, noises from the mechanical actuation, and wires obstructing natural handling.

Interestingly, some users reported the fast response time and high accuracy of the mechanical actuation was distracting. For instance, Participant 16 mentioned that: "In certain motions, it felt like whatever was moving inside of the system had to move incredibly aggressively in order to mimic the expected motion. This would cause weird movements of the system when adjusting." This in fact is the result of performing a sudden action with low viscosity simulated liquid. The simulated particles for this liquid lack high cohesion which causes particles to move around suddenly. SWISH in return responds quickly to this movement and follows the path of the CoG.

\section{LIMITATIONS AND FUTURE WORK}

\section{Electronic component limitations}

Our user study identified multiple limitations, revealing opportunities for further development. For some actions, SWISH's 
Table 2. Questionnaire statements and their relationship to VRUSE

\begin{tabular}{|c|c|c|}
\hline $\begin{array}{l}\text { Relationship } \\
\text { to VRUSE }\end{array}$ & Statement & $\begin{array}{l}\text { Mean } \\
\pm \text { SD }\end{array}$ \\
\hline Q. 8 & $\begin{array}{l}\text { SWISH tactile system was intuitive } \\
\text { to use for tasks of this study }\end{array}$ & $\begin{array}{c}4.38 \\
\pm 0.58\end{array}$ \\
\hline Q. 9 & $\begin{array}{l}\text { I would have preferred an alternative } \\
\text { tactile device }\end{array}$ & $\begin{array}{c}2.58 \\
\pm 0.72\end{array}$ \\
\hline Q. 10 & $\begin{array}{l}\text { SWISH tactile system's response } \\
\text { to my input was acceptable }\end{array}$ & $\begin{array}{c}4.21 \\
\pm 0.78\end{array}$ \\
\hline Q. 11 & $\begin{array}{l}\text { I found SWISH tactile system to be } \\
\text { too sensitive to use }\end{array}$ & $\begin{array}{c}2.42 \\
\pm 0.78\end{array}$ \\
\hline Q. 19 & $\begin{array}{l}\text { Visual feedback that I could see through } \\
\text { the headset was adequate }\end{array}$ & $\begin{array}{c}4.00 \\
\pm 0.78\end{array}$ \\
\hline Q. 20 & $\begin{array}{l}\text { Overall I would rate the SWISH tactile } \\
\text { system as a suitable VR input device }\end{array}$ & $\begin{array}{l}4.17 \\
\pm 0.76\end{array}$ \\
\hline $\begin{array}{c}\text { N/A } \\
\text { (Short Answer) }\end{array}$ & $\begin{array}{l}\text { What is your overall impression of the } \\
\text { SWISH tactile system. For instance, does } \\
\text { it match what you visually expect from } \\
\text { interacting with the virtual fluid? }\end{array}$ & \\
\hline $\begin{array}{c}\text { N/A } \\
\text { (Short Answer) }\end{array}$ & $\begin{array}{l}\text { What were the strengths of } \\
\text { the SWISH tactile system? }\end{array}$ & \\
\hline $\begin{array}{c}\text { N/A } \\
\text { (Short Answer) }\end{array}$ & $\begin{array}{l}\text { What were the shortcomings } \\
\text { of the SWISH tactile system? }\end{array}$ & \\
\hline
\end{tabular}

wires (for motor power and Arduino control) obstructed natural interaction with the vessel. We plan to create fully wireless designs through lower power design, battery packs, and embedded microcontroller attachments. Furthermore, connection with the Bluetooth module was unreliable and caused tens of milliseconds of latency. In the future, we will employ WiFibased microcontroller modules to avoid these issues.

\section{Audio for multi-modal fluid interactions}

Users commented that motor sounds were mildly distracting. We are exploring methods to dampen motor noises, including housing insulation and enclosures for the motor actuation. Furthermore, users commented that their experience with real fluids led them to expect sloshing sounds along with the visual and haptic experiences. We plan to look into audio synthesis to generate fluid sounds upon fluid particle interactions.

\section{Reshaped Miniaturization}

The SWISH enclosure can be redesigned to allow for multiple grip conditions and one-handed interaction. This can include pivot handles (such as on a pail bucket), or fixed handles (such as on the side of a coffee mug). We also plan to reshape and resize SWISH to match familiar vessels in chemistry labs. This will involve a re-selection of miniature motors that are capable of moving an adequately sized active mass around to generate the SWISH perceptions. Such investigation will significantly broaden the scope of utilization of SWISH actuation.

\section{CONCLUSION}

In this paper we presented SWISH, a novel haptic mechanism capable of providing believable tactile sensations of handling fluids in virtual environment. We design our SWISH actuation mechanism to move the vessel's CoG with $3 \mathrm{DoF}$ in real-time. Our end-to-end integration provides a robust and responsive system that is adaptive to simulated fluid dynamics. We evaluated SWISH's performance quantitatively and qualitatively and found evidence that using SWISH outperforms static passive proxies, towards emulating real fluids. The difficulties associated with handling real liquids - especially their lack of programmable capabilities and risk of spillage - encourage further research in this mostly unexplored field of creating tangibly reactive fluid vessels. Drawing on our promising results, we plan to explore ways to miniaturize the SWISH mechanism and improve its adaptivity to a wider range of use cases.

\section{ACKNOWLEDGMENTS}

We appreciate early support from the ASU Herberger Research Initiative seed grant and the School of Arts, Media and Engineering (AME) at ASU for Fabrication Lab support. We thank Linda Nguyen for figure assistance and Byron Lahey for project ideation. This material is based upon work supported by the National Science Foundation under Grant No. 1917912.

\section{APPENDIX FIGURES}

These figures show the motor command (orange dots) and position (blue line) for the radial, azimuthal, and height motors for liquids with low viscosity (left) and high viscosity (right).
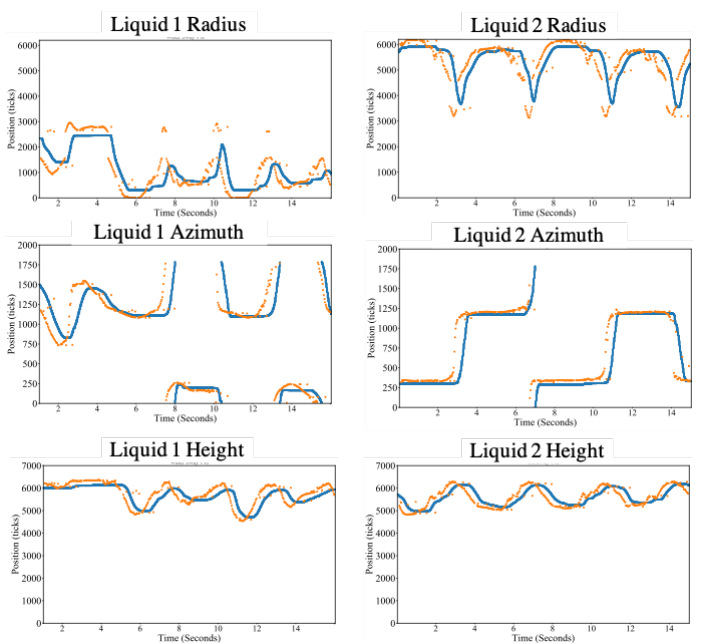

Figure 11. Sway action
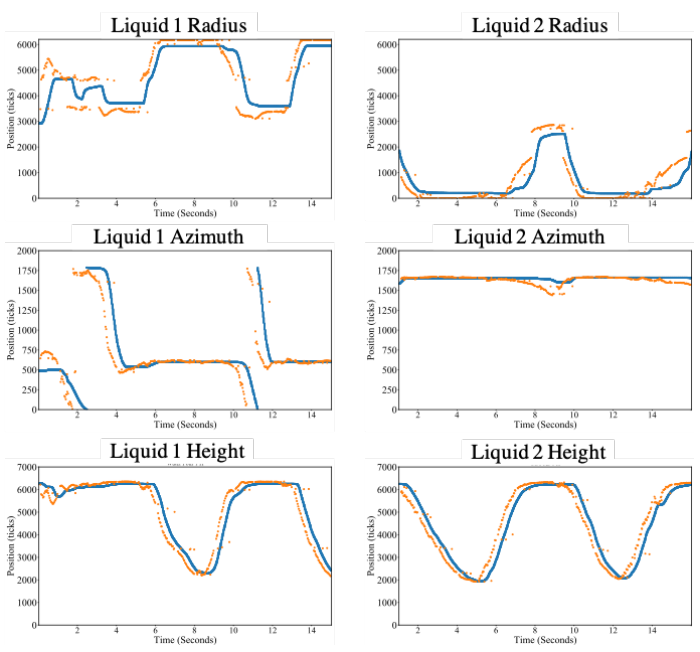

Figure 12. Pour action 


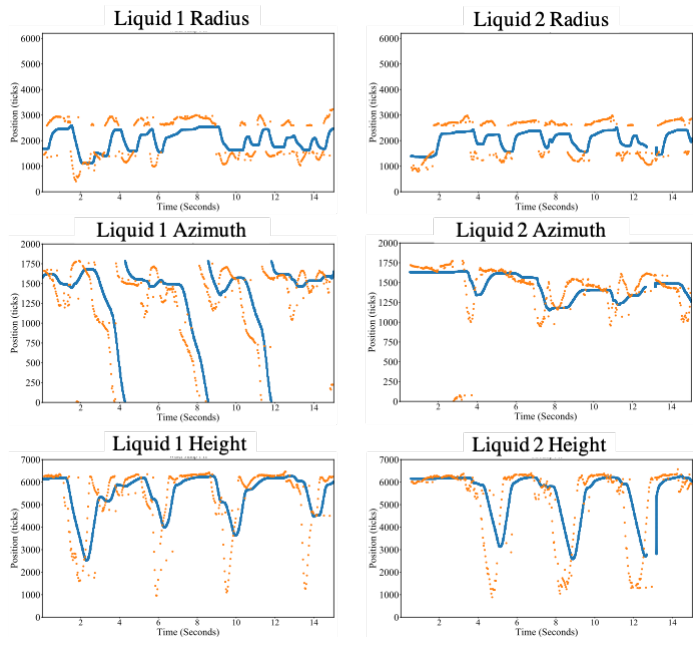

Figure 13. Jump action

\section{REFERENCES}

[1] Bruno Araujo, Ricardo Jota, Varun Perumal, Jia Xian Yao, Karan Singh, and Daniel Wigdor. 2016. Snake Charmer: Physically Enabling Virtual Objects. In Proc. of the TEI'16: Tenth International Conference on Tangible, Embedded, and Embodied Interaction. ACM, 218-226.

[2] Karl J Astrom. 1995. PID controllers theory, design, and tuning, 2nd edition (2nd ed ed.). S.l.]. http://portal. igpublish.com/iglibrary/search/ISAB0000021.html

[3] Hrvoje Benko, Christian Holz, Mike Sinclair, and Eyal Ofek. 2016. Normaltouch and texturetouch: High-fidelity $3 \mathrm{~d}$ haptic shape rendering on handheld virtual reality controllers. In Proc. of the 29th Annual Symposium on User Interface Software and Technology. ACM, 717-728.

[4] Inrak Choi, Elliot W Hawkes, David L Christensen, Christopher J Ploch, and Sean Follmer. 2016. Wolverine: A wearable haptic interface for grasping in virtual reality. In Proc. Intelligent Robots and Systems (IROS), 2016 IEEE/RSJ International Conference on. IEEE, 986-993.

[5] Gabriel Cirio, Maud Marchal, Miguel A Otaduy, and Anatole Lécuyer. 2013. Six-oof haptic interaction with fluids, solids, and their transitions. In Proc. World Haptics Conference (WHC), 2013. IEEE, 157-162.

[6] Heather Culbertson, Julie M Walker, and Allison M Okamura. 2016. Modeling and design of asymmetric vibrations to induce ungrounded pulling sensation through asymmetric skin displacement. In Proc. Haptics Symposium (HAPTICS), 2016 IEEE. IEEE, 27-33.

[7] Eisuke Fujinawa, Shigeo Yoshida, Yuki Koyama, Takuji Narumi, Tomohiro Tanikawa, and Michitaka Hirose. 2017. Computational design of hand-held VR controllers using haptic shape illusion. In Proceedings of the 23rd ACM Symposium on Virtual Reality Software and Technology. ACM, 28.
[8] Xiaochi Gu, Yifei Zhang, Weize Sun, Yuanzhe Bian, Dao Zhou, and Per Ola Kristensson. 2016. Dexmo: An inexpensive and lightweight mechanical exoskeleton for motion capture and force feedback in VR. In Proc. of the 2016 CHI Conference on Human Factors in Computing Systems. ACM, 1991-1995.

[9] Daniel Harley, Alexander Verni, Mackenzie Willis, Ashley Ng, Lucas Bozzo, and Ali Mazalek. 2018. Sensory VR: Smelling, Touching, and Eating Virtual Reality. In Proc. of the Twelfth International Conference on Tangible, Embedded, and Embodied Interaction. ACM, 386-397.

[10] K. Hinkelmann and O. Kempthorne. 2007. Design and Analysis of Experiments, Volume 1: Introduction to Experimental Design. Wiley. https://books. google. com/books?id=T3wWj2kVYZgC

[11] Roy S. Kalawsky. 1999. VRUSE—a computerised diagnostic tool: for usability evaluation of virtual/synthetic environment systems. Applied Ergonomics 30, 1 (1999), 11 - 25. DOI :

http://dx.doi.org/https:

//doi.org/10.1016/S0003-6870(98)00047-7

[12] Miles Macklin and Matthias Müller. 2013. Position Based Fluids. ACM Trans. Graph. 32, 4 (2013), 104:1-104:12. DOI:

http://dx.doi.org/10.1145/2461912 . 2461984

[13] Miles Macklin, Matthias Müller, Nuttapong Chentanez, and Tae-Yong Kim. 2014. Unified Particle Physics for Real-time Applications. ACM Trans. Graph. 33, 4 (2014), 153:1-153:12. DOI: http://dx.doi.org/10.1145/2601097.2601152

[14] Thomas H Massie, J Kenneth Salisbury, and others. 1994. The phantom haptic interface: A device for probing virtual objects. In Proc. of the ASME winter annual meeting, symposium on haptic interfaces for virtual environment and teleoperator systems, Vol. 55. Citeseer, 295-300.

[15] Kouta Minamizawa, Souichiro Fukamachi, Hiroyuki Kajimoto, Naoki Kawakami, and Susumu Tachi. 2007. Gravity grabber: wearable haptic display to present virtual mass sensation. In Proc. ACM SIGGRAPH 2007 emerging technologies. ACM, 8.

[16] Anne M Murray, Roberta L Klatzky, and Pradeep K Khosla. 2003. Psychophysical characterization and testbed validation of a wearable vibrotactile glove for telemanipulation. Presence: Teleoperators \& Virtual Environments 12, 2 (2003), 156-182.

[17] Ryuma Niiyama, Lining Yao, and Hiroshi Ishii. 2014. Weight and volume changing device with liquid metal transfer. In Proc. of the 8th International Conference on Tangible, Embedded and Embodied Interaction. ACM, 49-52. 
[18] Adalberto L Simeone, Eduardo Velloso, and Hans Gellersen. 2015. Substitutional reality: Using the physical environment to design virtual reality experiences. In Proc. of the 33rd Annual ACM Conference on Human Factors in Computing Systems. ACM, 3307-3316.

[19] Colin Swindells, Alex Unden, and Tao Sang. 2003. TorqueBAR: an ungrounded haptic feedback device. In Proc. of the 5th international conference on Multimodal interfaces. ACM, 52-59.

[20] Richard Q Van der Linde, Piet Lammertse, Erwin Frederiksen, and B Ruiter. 2002. The HapticMaster, a new high-performance haptic interface. In Proc. Eurohaptics. 1-5.

[21] Mauricio Vines, Javier Mora, and Won-Sook Lee. 2009. Haptic display of 3D liquids for interactive applications. In Proc. Games Innovations Conference, 2009. ICE-GIC 2009. International IEEE Consumer Electronics Society's. IEEE, 140-148.

[22] Julie Walker, Heather Culbertson, Michael Raitor, and Allison Okamura. 2017. Haptic orientation guidance using two parallel double-gimbal control moment gyroscopes. IEEE transactions on haptics (2017).

[23] Kyle N Winfree, Joseph M Romano, Jamie Gewirtz, and Katherine J Kuchenbecker. 2010. Control of a high fidelity ungrounded torque feedback device: The iTorqU 2.1. In Proc. Robotics and Automation (ICRA), 2010 IEEE International Conference on. IEEE, 1347-1352.
[24] Burton Woodruff and Harry Helson. 1965. Torque: A New Dimension in Tactile-Kinesthetic Sensitivity. The American Journal of Psychology 78, 2 (1965), 271-277. DOI : http://dx.doi .org/10.2307/1420500

[25] Vibol Yem, Ryuta Okazaki, and Hiroyuki Kajimoto. 2016. FinGAR: combination of electrical and mechanical stimulation for high-fidelity tactile presentation. In Proc. ACM SIGGRAPH 2016 Emerging Technologies. ACM, 7.

[26] André Zenner. 2016. Investigating weight distribution in virtual reality proxy interaction. Universität des Saarlandes, Masterarbeit (2016).

[27] Andre Zenner and Antonio Krüger. 2017. Shifty: A Weight-Shifting Dynamic Passive Haptic Proxy to Enhance Object Perception in Virtual Reality. IEEE Transactions on Visualization and Computer Graphics 23, 4 (2017), 1285-1294.

[28] Jiachao Zhang, Shunpei Yuasa, Shinji Fukuma, and Shin-Ichiro Mori. 2016. A real-time GPU-based coupled fluid-structure simulation with haptic interaction. In Proc. 2016 IEEE/ACIS 15th International Conference on Computer and Information Science (ICIS). IEEE, 1-6.

[29] MA Zhou and Pinhas Ben-Tzvi. 2015. RML glove-An exoskeleton glove mechanism with haptics feedback. IEEE/ASME Transactions on Mechatronics 20, 2 (2015), 641-652. 